Wilfrid Laurier University

Scholars Commons @ Laurier

Physics and Computer Science Faculty

Publications

Physics and Computer Science

2014

\title{
Enhanced Capillary Rise of Wetting Liquids in Reduced Gravitational Shielding Under Microgravity Conditions
}

\author{
George D. Zouganelis \\ Bournemouth and Poole College Health and Medical Sciences \\ loannis Gkigkitzis \\ East Carolina University \\ Ioannis Haranas \\ Wilfrid Laurier University, iharanas@wlu.ca
}

Follow this and additional works at: https://scholars.wlu.ca/phys_faculty

Part of the Computer Sciences Commons, and the Physics Commons

\section{Recommended Citation}

Zouganelis, G.D., I. Gkigkitzis, I. Haranas, 2014. Enhanced Capillary Rise of Wetting Liquids in Reduced Gravitational Shielding Under Microgravity Conditions. Physics International 5(2): 140-151. DOI: 10.3844/ pisp.2014.140.151

This Article is brought to you for free and open access by the Physics and Computer Science at Scholars Commons @ Laurier. It has been accepted for inclusion in Physics and Computer Science Faculty Publications by an authorized administrator of Scholars Commons @ Laurier. For more information, please contact scholarscommons@wlu.ca. 


\title{
ENHANCED CAPILLARY RISE OF WETTING LIQUIDS IN REDUCED GRAVITATIONAL SHIELDING UNDER MICROGRAVITY CONDITIONS
}

\author{
${ }^{1}$ George D. Zouganelis, ${ }^{2}$ Ioannis Gkigkitzis and ${ }^{3}$ Ioannis Haranas \\ ${ }^{1}$ Bournemouth and Poole College Health and Medical Sciences \\ North Road, Poole, Dorset, BH14 OLS, UK \\ ${ }^{2}$ Departments of Mathematics and Biomedical Physics, \\ East Carolina University, 124 Austin Building, East Fifth Street Greenville NC 27858-4353, USA \\ ${ }^{3}$ Department of Physics and Astronomy, \\ York University 4700 Keele Street, Toronto, Ontario, M3J 1P3, Canada
}

Received 2014-05-04; Revised 2014-06-16; Accepted 2014-06-21

\begin{abstract}
We study the capillary rise of wetting in liquids by slightly modifying Ponomarenko's result, a recently derived and observed $t^{1 / 3}$ law, without omitting the corresponding gravity term and therefore we find $h_{\text {new }}$ $(t) \cong 0.9085 h_{P o n}(t)$ instead, which corresponds to a $9 \%$ difference. Furthermore, in order to examine the effect of corrected gravity, we extend the result on the surface of a planetary body by correcting the gravitational acceleration for its oblateness coefficient and rotation. We find that experiments that take place on the equator result in highest capillary heights, than those at mid latitudes and the poles. Similarly, analyzing the effect of reduced gravitational shielding on the capillary rise under conditions of microgravity in experiments aboard orbiting spacecraft, we find that equatorial circular orbits exhibit the highest capillary heights, where equatorial elliptical orbits of large eccentricities exhibit the smallest capillary heights. Finally, we calculate the rate of change of the meniscus height in the time domain, for the different laboratory conditions on the surface of the Earth and in space and as example. As an example we say that laboratory sites at the equator will exhibit larges time rates of change for the meniscus height. Similarly, for experiments above a spacecraft we find that circular equatorial orbits exhibit the highest time rates of change, where elliptical orbits exhibit smaller time rates that reduce as the eccentricity increases.
\end{abstract}

Keywords: Capillary Effect Rise, Capillary Height, Wetting, Microgravity, Gravitational Acceleration, Oblateness

\section{INTRODUCTION}

Capillary rise is used in the study of the performance of low-pressure plasma treatments with different gases on synthetic fabrics in order to improve wettability (Ferrero, 2003), in the study of the shape of an advancing interface in a liquid gas system (Hoffman, 1983), as a mechanism of water penetration into building materials (Karoglou et al., 2005), for the characterization of the structure of porous media (Marmur, 2003), in the study the shape of oil droplets for lubrication applications (Tanner, 1979), for the determination of the wettability of powders (Galet et al., 2010), among many other applications. In addition, in plant systems, four forces are involved in water transport from the roots, through the xylem elements to the roots. Water is transpired through the leaves pulling water up through the xylem of the plant utilizing this way the energy of evaporation and tensile strength of water. The movement of water from the roots to the leaves is driven by a combination of

(C) 2014 George D. Zouganelis, loannis Gkigkitzis and loannis Haranas. This open access article is distributed under a Creative Commons Attribution (CCBY) 3.0 license which permits unrestricted use, distribution, and reproduction in any medium, provided the original work is properly credited. DOI: $10.3844 /$ pisp.2014.140.151 
adhesion, cohesion and tension forces through capillary formations known as xylem structures (Nobel, 1999). Capillary action effects are also of significance for improvement of appropriate porous media involved in plant growth experiments in space (Jones, 1998; 1999). Furthermore, the capillary rise of liquids plays an important role in wetting phenomena and liquid motions through porous media especially under microgravity because the capillary rise that is suppressed under normal gravity become dominant under conditions of microgravity (gravitational shielding of capillary effects) (Kaneki et al., 2000). High altitude aircraft parabolic flights (European Space Agency Caravelle aircraft) and an orbiting space shuttle (NASA discovery mission STS-91) have been used to conduct capillary flow experiments in porous media, as part of a broader program aimed at a better understanding of mechanisms involved in processes designed to recover oil and gas from petroleum reservoirs with the use of different fluids it has been observed that there is a masking influence of normal gravity on capillary flows (Schramm et al., 2003; Smirnov et al., 2004). Moreover capillary rise phenomena have direct applications to a number of problems associated with fluid handling in space vehicles of various kinds (Ostrach, 1982; Schramm et al., 2003). The enhanced apparent capillary phenomena such as the capillary rise, that have been observed under conditions of corrected gravity merit a further theoretical investigation to existing experimental models. Our study is based on the experimental set-up and derived and observed model introduced in (Ponomarenko et al., 2011) where two solid rods made of Plexiglas are pressed together by regularly spaced threaded rods and the capillary rise of silicon oil (the wetting liquid contained in a Petri dish whose vertical position is controlled by a Micro-Controle translation table) is observed through the cylinders and the location of the liquid front $h(t)$ and its time evolutions is determined. The background laboratory in our study will be (a) on the surface of a planetary body and (b) inside an orbiting spacecraft.

The organ model consists of a collection of juxtaposed tubes of decreasing diameters as they approach the corner and $h_{r}(r, t)$ is the location of the front in the tube of radius $r$ at time $t$. Based on Stokes' equation the driving capillary pressure gradient $\frac{\gamma}{r h_{r}}$ is balanced by both the force of gravity $\rho g$ and the viscous friction based on the velocity of the front $h_{r}$ and the location of the front is then given by the equation (Ponomarenko et al., 2011):

$$
h_{r}(r, t) \cong \sqrt{\frac{2 r \gamma t}{\eta}}-\frac{\rho g r^{2}}{\eta} t
$$

Taking the first derivative with respect to $r$ of Equation 1 and equating to zero we can obtain the radius of the leading meniscus and therefore we have that:

$$
\left(\frac{\partial h_{r}}{\partial r}\right)_{r=r_{L}} \cong \frac{\gamma t}{\sqrt{2 \eta r \gamma t}}-\frac{2 g \rho r t}{\eta}=0
$$

Solving the equation below Equation 2 for we $r$, we obtain the leading radius of the meniscus $r_{L}$ to be:

$r_{L} \cong\left(\frac{\gamma \eta}{8 g^{2} \rho^{2} t}\right)^{1 / 3}$

Next, substituting Equation 3 in (1) without omitting $\rho g r^{2} t / \eta$ as in (Ponomarenko et al., 2011) we find that the capillary height rises as a function of time $t$ and $h(t)$ is given by a slightly different expression that involves numerical factor $(3 / 4)^{1 / 3}$ $\approx 0.9085$ and therefore we find that:

$$
h_{\text {new }}(t) \cong h_{y r}\left(r_{L}, t\right) \cong\left(\frac{3 \gamma^{2} t}{4 g \rho \eta}\right)^{1 / 3} \cong 0.90856 h_{\text {Pon }}(t)
$$

\subsection{The Gravitational Acceleration at Orbital Point and the Action of Gravity in Capillaries}

In our effort to study the effect of gravity on capillaries in altered gravity environments let us consider the acceleration of gravity $g$ at the surface of a spherical body as well as at the orbital altitude of the spacecraft in which a capillary experiment is taking place under controlled conditions. At a radial distance $r$ of the spacecraft from the center of the planetary body we have that the corrected acceleration is given by the sum of three different components namely:

$$
g_{\text {tot }}(r)=g_{\text {cen }}(r)+g_{J_{2}}(r)+g_{\text {rot }}(r)
$$

where, $g_{c e n}(r)$ represents the central part of the gravitational acceleration, $g_{J 2}(r)$ is the correction of the gravitational acceleration due to the oblateness of 
the planetary body and finally $g_{r o t}(r)$ the contribution in the gravitational potential due the rotation of the planetary body. Furthermore following (Haranas and Harney, 2008; Haranas et al., 2012) we write the magnitude of the acceleration of gravity to be:

$$
g_{t o t}(a, e, f, \omega, i)=\frac{G M}{r^{2}}-\frac{3 G M R^{2} J_{2}}{2 r^{4}}\left(3 \sin ^{2} \varphi-1\right)-\omega^{2} r \cos ^{2} \varphi
$$

where, $M$ is the mass of the spherical body, $J_{2}$ is the zonal harmonic coefficient that describes the oblateness of the Earth. Zonal harmonics are bands of latitude, whose boundaries are the roots of Legendre polynomial (Vallado, 2001). This particular gravitational harmonic coefficient is a result of the Earth's shape and is about 1000 times larger than the next harmonic coefficient $J_{3}$ and its value is equal to $J_{2}=-0.0010826260$ (Vallado, 2001). Given that the radial distance of the spacecraft is given by Equation 7:

$r=\frac{a\left(1-e^{2}\right)}{(1+e \cos f)}$

Equation 6 becomes a function of the spacecraft orbital elements which for an elliptical orbit it takes the form:

$$
\begin{aligned}
& g_{\text {tot }}=\frac{G M_{E}(1+e \cos f)^{2}}{a^{2}\left(1-e^{2}\right)^{2}}- \\
& \frac{3 G M_{E} R_{E}^{2} J_{2}(1+e \cos f)^{4}}{2 a^{4}\left(1-e^{2}\right)^{4}}\left(\sin ^{2} i \sin ^{2} f-1\right)
\end{aligned}
$$

where, $a$ is the semi-major axis of the orbiting spacecraft, $i$ is its orbital inclination and $f$ is its true anomaly and we have also used the relation $\sin \phi=\sin I$ $\sin (\omega+f)$, which is a standard transformation of celestial mechanics relating planetocentric latitude $\varphi$, or colatitude $\theta$, to the inclination $i$, argument of the perigee $\omega$ and the true anomaly $f$ of the orbiting spacecraft.

\subsection{Capillary Rise of Wetting Liquids on the Surface of a Spherical Body}

First let us consider a capillary experiment that takes place on the surface of the Earth $r=R_{E}$. In this case from Equation 6 we obtain the following expression for the acceleration of gravity corrected for the oblateness and the rotation of the Earth on the Earth's surface and therefore the gravitational acceleration $g_{s}$ becomes:

$$
g_{s}=\frac{G M_{E}}{R_{E}^{2}}-\frac{3 G M_{E} J_{2}}{2 R_{E}^{2}}\left(3 \sin ^{2} \varphi-1\right)-R_{E} \omega_{E}^{2} \cos ^{2} \varphi
$$

Furthermore, thinking the Earth to be an ellipsoid of revolution we can write that (Kaula, 2000) Equation 10:

$$
\begin{aligned}
& R_{E}=R_{e q}\left(1-\left(f^{\prime}+\frac{3}{2} f^{\prime 2}\right) \sin ^{2} \varphi_{E}+\frac{3}{2} f^{\prime 2} \sin ^{4} \varphi_{E}-\ldots\right) \\
& » R_{e q}\left(1-f^{\prime} \sin ^{2} \varphi_{E}\right)
\end{aligned}
$$

where, $\varphi$ is the planetocentric latitude and $f^{\prime}$ is the Earth's flattering that it is given by Equation 11:

$f^{\prime}=\frac{R_{e q}-R_{p o l}}{R_{e q}}=\frac{3 J_{2}}{2}+\frac{R_{E}^{3} \omega_{E}^{2}}{2 G M_{E}}$

where, $R_{e q}$ and $R_{p o l}$ is the Earth's equatorial and polar radii and therefore. Equation 9 becomes:

$$
\begin{aligned}
& g_{s}=\frac{G M_{E}}{R_{e q}^{2}\left(1-f^{\prime} \sin ^{2} \varphi\right)^{2}} \\
& -\frac{3 G M_{E} J_{2}}{2 R_{e q}^{2}\left(1-f^{\prime} \sin ^{2} \varphi\right)^{2}}\left(3 \sin ^{2} \varphi-1\right)-R_{e q}\left(1-f^{\prime} \sin ^{2} \varphi\right) \omega_{E}^{2} \cos ^{2} \varphi
\end{aligned}
$$

On the surface of the Earth and for the geocentric latitude $\phi=0^{\circ}, 45^{\circ}, 90^{\circ}$ Equation 12 results in the following expressions for the gravitational acceleration Equation 13 to 15 :

$$
\begin{aligned}
& g_{j=0}=\frac{G M_{E}}{R_{e q}^{2}}+\frac{3 G M_{E} J_{2}}{2 R_{e q}^{2}}-R_{e q} \omega_{E}^{2}, \\
& g_{j=45}=\frac{G M_{E}}{R_{e q}^{2}\left(1-f^{\prime}\right)^{2}}+\frac{3 G M_{E} J_{2}}{4 R_{e q}^{2}\left(1-\frac{f^{\prime}}{2}\right)^{2}}-\frac{1}{2}\left(1-\frac{f^{\prime}}{2}\right) R_{e q} \omega_{E}^{2} \\
& g_{j=90}=\frac{G M_{E}}{R_{e q}^{2}\left(1-f^{\prime}\right)^{2}}+\frac{3 G M_{E} J_{2}}{R_{e q}^{2}\left(1-f^{\prime}\right)^{2}}
\end{aligned}
$$

Therefore, substituting in Equation 4 we obtain the following expressions for the capillary height rise for the geocentric latitudes of $\varphi=0^{\circ}, 45^{\circ}, 90^{\circ}$ given above and therefore we respectively obtain:

$$
h(t)=h_{\gamma r}\left(r_{L}, t\right)=\left(\frac{3 \gamma^{2} t}{4 \rho \eta}\left(\frac{G M_{E}}{R_{e q}^{2}}+\frac{3 G M_{E} J_{2}}{2 R_{e q}^{2}}-R_{e q} \omega_{E}^{2}\right)^{-1}\right)^{1 / 3}
$$




$$
\begin{aligned}
& h(t)=h_{\gamma r}\left(r_{L}, t\right) \\
& =\left(\frac { 3 \gamma ^ { 2 } t } { 4 \rho \eta } \left(\begin{array}{l}
\frac{G M_{E}}{R_{e q}^{2}\left(1-f^{\prime}\right)^{2}} \\
\left.\left.+\frac{3 G M_{E} J_{2}}{4 R_{e q}^{2}\left(1-\frac{f^{\prime}}{2}\right)^{2}}-\frac{1}{2}\left(1-\frac{f^{\prime}}{2}\right) R_{e q} \omega_{E}^{2}\right)^{-1}\right)^{1 / 3} \\
h(t)=h_{\gamma r}\left(r_{L}, t\right)=\left(\frac{3 \gamma^{2} t}{4 \rho \eta}\left(\frac{G M_{E}}{R_{e q}^{2}\left(1-f^{\prime}\right)^{2}}+\frac{3 G M_{E} J_{2}}{R_{e q}^{2}\left(1-f^{\prime}\right)^{2}}\right)^{-1}\right)^{1 / 3}
\end{array}\right.\right.
\end{aligned}
$$

\subsection{The $\mathbf{J}_{2}$ Harmonic and Flattering Resulting from a Surface Earth Capillary Experiments}

Solving Equation 16 to 18 we obtain an expression for the $J_{2}$ spherical harmonic of the Earth, as a function of the experimental parameters involved in a capillary experiment that is taking place on the surface of the Earth and therefore we respectively find that Equation 19 to 21:

$$
\begin{aligned}
& J_{2}=-\frac{1}{6}\left(4-\frac{\left(3 \gamma^{2} t+4 \omega_{E}^{2} h^{3}(t) \eta \rho\right)}{G M_{E} \rho \eta h^{3}(t)} R_{e q}^{2}\right) \\
& J_{2}=\frac{3\left(2-f^{\prime}\right)^{2} R_{E}^{2} \gamma^{2} t+h^{3}(t)\left(16 G M_{E}-\left(2-f^{\prime}\right)^{3} R_{E}^{3} \omega_{E}^{2}\right) \eta \rho}{12 G M_{E} \rho \eta h^{3}(t)}
\end{aligned}
$$

$J_{2}=\frac{1}{3}+\frac{\left(1-f^{\prime}\right)^{2} R_{e q}^{2}}{4 G M_{E} \rho \eta h^{3}(t)} t$

Similarly we can also obtain analytical solution for the flattering of a spherical body if the height $\mathrm{h}(\mathrm{t})$ of a capillary experiment can be measured from the cases of $\varphi=45^{\circ}, 90^{\circ}$. Here we only give the solution that results from $\varphi=90^{\circ}$ geocentric latitude and therefore we can obtain the following expression for the planetary flattering to be Equation 22 to 24 :

$$
\begin{gathered}
-R_{e q}^{2} \gamma^{2} t+\frac{R_{e q}^{2} \gamma^{4} t^{2}}{\left[2 \sqrt{Q}+R_{e q}^{6}\left(H-\gamma^{6} t^{3}\right)\right]^{\frac{1}{3}}} \\
f^{\prime}=\frac{+\left[2 \sqrt{Q}+R_{e q}^{6}\left(H-\gamma^{6} t^{3}\right)\right]^{\frac{1}{3}}}{h^{3}(t) R_{e q}^{3} \eta \rho}
\end{gathered}
$$

Where:

$$
H=2 G M_{e q}\left(4-3 J_{2}\right) h^{9}(t) \omega_{E}^{4} \eta^{3} \rho^{3}
$$

$$
\begin{aligned}
& H=G M_{E} R_{e q}^{22}\left(3 J_{2}-4\right) h^{9}(t) \omega_{E}^{4} \eta^{3} \rho^{3} \\
& \left(\gamma^{6} t^{3}+G M_{E} h^{9}(t) \eta^{3} \rho^{3}\left(3 J_{2}-4\right)\right)
\end{aligned}
$$

Finally when $\varphi=45^{\circ}$ we obtain that Equation 25:

$$
f^{\prime}=1 \pm 2 \sqrt{\frac{G M_{e q}\left(1-3 J_{2}\right) h^{3}(t) \eta \rho}{3 R_{e q}^{2} \gamma^{2} t}}
$$

\subsection{Capillary Height in Altered Gravity Conditions}

Next let us consider a capillary experiment that is taking place in altered gravity conditions and to be more specific in a spacecraft orbiting a spherical body. First, let us consider a circular orbits $e=0$ with various inclinations i.e., $i=0^{\circ}, 45^{\circ}, 90^{\circ}$. Using Equation 8 we obtain that the corresponding capillary heights become:

$h(t)=h_{\gamma r}\left(r_{L}, t\right)=\left(\frac{\gamma^{2} t}{\rho \eta}\left(\frac{G M_{E}}{a^{2}}+\frac{3 G M_{E} R_{e q}^{2} J_{2}}{2 a^{4}}\right)^{-1}\right)^{1 / 3}$

Similarly Equation 26 to 31:

$h(t)=h_{\gamma r}\left(r_{L}, t\right)=$

$\left(\frac{\gamma^{2} t}{\rho \eta}\left(\frac{G M_{E}}{a^{2}}+\frac{3 G M_{E} R_{e q}^{2} J_{2}}{2 a^{4}}\left(\frac{3 \sin ^{2} f}{2}-1\right)\right)^{-1}\right)^{1 / 3}$

And finally:

$h(t)=h_{\gamma r}\left(r_{L}, t\right)=$

$\left(\frac{\gamma^{2} t}{\rho \eta}\left(\frac{G M_{E}}{a^{2}}+\frac{3 G M_{E} R_{e q}^{2} J_{2}}{2 a^{4}}\left(\frac{3 \sin ^{2} f-1}{2}\right)\right)^{-1}\right)^{1 / 3}$

Similarly, for elliptical orbits we obtain that:

$$
\begin{aligned}
& h(t)=h_{\gamma r}\left(r_{L}, t\right)= \\
& \left(\frac{\gamma^{2} t}{\rho \eta}\left(\frac{G M_{E}(1+e \cos f)}{a^{2}\left(1-e^{2}\right)^{2}}+\frac{3 G M_{E} R_{e q}^{2} J_{2}(1+e \cos f)^{4}}{2 a^{4}\left(1-e^{2}\right)^{4}}\right)^{-1}\right)^{1 / 3} \\
& h(t)=h_{\gamma r}\left(r_{L}, t\right)= \\
& \left(\frac{\gamma^{2} t}{\rho \eta}\left(\frac{\frac{G M_{E}(1+e \cos f)}{a^{2}\left(1-e^{2}\right)^{2}}+}{\frac{3 G M_{E} R_{e q}^{2} J_{2}(1+e \cos f)^{4}}{2 a^{4}\left(1-e^{2}\right)^{4}}\left(\frac{3 \sin ^{2} f}{2}-1\right)}\right)^{-1}\right)^{1 / 3}
\end{aligned}
$$




$$
\begin{aligned}
& h(t)=h_{\gamma r}\left(r_{L}, t\right)= \\
& \left(\frac{\gamma^{2} t}{\rho \eta}\left(\begin{array}{l}
\frac{G M_{E}(1+e \cos f)}{a^{2}\left(1-e^{2}\right)^{2}}+ \\
\frac{3 G M_{E} R_{e q}^{2} J_{2}(1+e \cos f)^{4}}{2 a^{4}\left(1-e^{2}\right)^{4}}\left(\frac{3 \sin ^{2} f-1}{2}\right)
\end{array}\right)^{-1}\right)^{1 / 3}
\end{aligned}
$$

\subsection{The $\mathbf{J}_{2}$ Harmonic and Flattering Resulting from Space Experiments and the Rate of Change of Meniscus Height}

Next, let us consider a capillary experiment that is taking place in altered gravity conditions and to be more specific in a spacecraft orbiting a spherical body of oblateness $J_{2}$. First, let us consider a circular orbits $e=0$ with various inclinations i.e., $i=0^{\circ}, 45^{\circ}, 90^{\circ}$. Using Equation 8 we obtain that the corresponding capillary heights become Equation 32 to 34:

$$
\begin{aligned}
& J_{2}=\frac{a^{2}}{6 R_{E}^{2}}\left(-4+\frac{3 a^{2} \gamma^{2} t}{G M_{E} h^{3}(t) \eta \rho}\right) \\
& J_{2}=\frac{3 a^{4} \gamma^{2} t-4 G M_{E} a^{2} h^{3}(t) \eta \rho}{9 G M_{E} h^{3}(t) R_{E}^{2} \eta \rho\left(\sin ^{2} u-\frac{2}{3}\right)} \\
& J_{2}=\frac{3 a^{4} \gamma^{2} t-4 G M_{E} a^{2} h^{3}(t) \eta \rho}{6 G M_{E} h^{3}(t) R_{E}^{2} \eta \rho\left(1-3 \sin ^{2} u\right)}
\end{aligned}
$$

where, for circular orbits the element of true anomaly cannot be defined and gets replaced with the argument of the latitude $u$ instead. Similarly for elliptical orbits we find that Equation 35 to 37 :

$$
\begin{gathered}
J_{2}=\frac{a^{2}\left(1-e^{2}\right)^{2}\left(\begin{array}{l}
3 a^{2}\left(1-e^{2}\right) \gamma^{2} t-4 G M_{E} h^{3} \\
(t) \rho \eta-4 e G M_{E} h^{3}(t) \cos f(2+e \cos f)
\end{array}\right)}{6 G M R_{e q}^{2} h^{3}(t) \rho \eta(1+e \cos f)^{4}} \\
J_{2}=-\frac{a^{2}\left(1-e^{2}\right)^{2}\left(\begin{array}{l}
3 a^{2}\left(1-e^{2}\right)^{2} \gamma^{2} t-4 G M_{E} h^{3}(t) \eta \rho- \\
4 e G M_{E} h^{3}(t) \eta \rho \cos f(2+e \cos f)
\end{array}\right)}{3 G M R_{e q}^{2} h^{3}(t) \rho \eta(1+e \cos f)^{4}\left(3 \sin ^{2} f-2\right)} \\
J_{2}=-\frac{a^{2}\left(1-e^{2}\right)^{2}\left(\begin{array}{l}
3 a^{2}\left(1-e^{2}\right) \gamma^{2} t-4 G M_{E} h^{3}(t) \eta \rho \\
-4 e G M_{E} h^{3}(t) \eta \rho \cos f(2+e \cos f)
\end{array}\right)}{6 G M R_{e q}^{2} h^{3}(t) \rho \eta(1+e \cos f)^{4}\left(3 \sin ^{2} f-1\right)}
\end{gathered}
$$

As a last step of our work we calculate the velocities at which of the meniscus height changing. Therefore on the surface of the Earth we obtain that Equation 38:

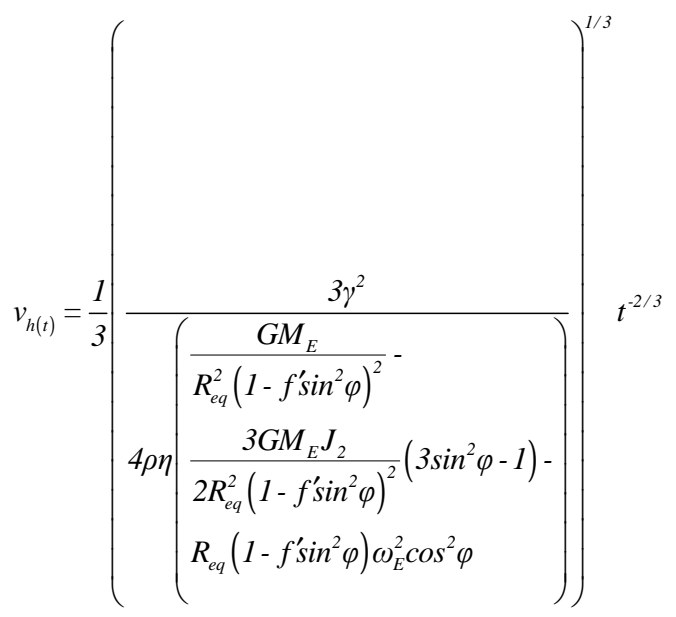

which for the particular latitudes $\varphi=0^{\circ}, 45^{\circ}, 90^{\circ}$ takes the form Equation 39 and 40:

$v_{h(t)}=\frac{1}{3}\left(\frac{3 \gamma^{2}}{4 \rho \eta}\left(\frac{G M_{E}}{R_{e q}^{2}}+\frac{3 G M_{E} J_{2}}{2 R_{e q}^{2}}-R_{e q} \omega_{E}^{2} \cos ^{2} \varphi\right)^{-1}\right)^{1 / 3} t^{-2 / 3}$

$v_{h(t)}=\frac{1}{3}\left(\frac{3 \gamma^{2}}{4 \rho \eta}\left(\frac{\frac{G M_{E}}{R_{e q}^{2}\left(1-f^{\prime}\right)^{2}}+}{\frac{3 G M_{E} J_{2}}{4 R_{e q}^{2}\left(1-\frac{f^{\prime}}{2}\right)^{2}}-\frac{1}{2}\left(1-\frac{f^{\prime}}{2}\right) R_{e q} \omega_{E}^{2}}\right)^{-1}\right)^{1 / 3} t^{-2 / 3}$

and finally Equation 41:

$v_{h(t)}=\frac{1}{3}\left(\frac{3 \gamma^{2}}{4 \rho \eta}\left(\frac{G M_{E}\left(1-3 J_{2}\right)}{R_{e q}^{2}\left(1-f^{\prime}\right)^{2}}\right)^{-1}\right)^{1 / 3} t^{-2 / 3}$

Next, let us proceed with the calculation of the velocity of the meniscus height in space and in a microgravity environment let us consider the acceleration of gravity at the orbital altitude of the spacecraft. Since the derivative $w . r$. $t$. to time $t$ should be taken, we write Equation 8 as a function of time $t$ making use of well known transformations of celestial 
mechanics. For that we use the relation between the true anomaly $f$ to the true anomaly $M$ (Murray and Dermott, 1999) Equation 42:

$$
\begin{aligned}
& f=f(n t) » M+2 e \sin M+\frac{5 e^{2}}{4} \sin 2 M=n t \\
& +2 e \sin (n t)+\frac{5 e^{2}}{4} \sin (2 n t) \ldots
\end{aligned}
$$

Using Equation 40 and substituting in Equation 8 and then Equation 8 into Equation 4 we obtain that Equation 43 and 44:

$$
h(t)=h_{\gamma r}\left(r_{L}, t\right)=\left(\frac{3 \gamma^{2} t}{4 g_{t o t}(n t) \rho \eta}\right)^{1 / 3}
$$

Where:

$$
\begin{aligned}
& g_{\text {tot }}(n t)=\frac{G M_{E}(1+e \cos (f(n t)))^{2}}{a^{2}\left(1-e^{2}\right)^{2}}- \\
& \frac{3 G M_{E} R_{E}^{2} J_{2}(1+e \cos f(n t))^{4}}{2 a^{4}\left(1-e^{2}\right)^{4}}\left(\sin ^{2} i \sin ^{2} f(n t)-1\right)
\end{aligned}
$$

where, $f(n t)$ is given by Equation 5 and where $n$ is the mean motion of the spacecraft in $\mathrm{rad} / \mathrm{s}$ and therefore the capillary meniscus velocity on a general elliptical orbit of eccentricity $e$ and semi-major axis $a$ becomes Equation 45 to 49 :

$$
v_{h(t)}=\frac{\left(\frac{\gamma^{2}}{\eta \rho Q_{1}}\left(1-\frac{Q_{2} t}{Q_{1}}\right)\right)}{6^{2 / 3} Q_{l}^{2 / 3} t^{2 / 3}}
$$

Where:

$$
\begin{aligned}
A & =n+2 n e \cos (n t)+\frac{5 n e^{2}}{2} \cos (2 n t) \\
B & =n t+2 e \cos (n t)+\frac{5 e^{2}}{2} \cos (2 n t) \\
Q_{1} & =\frac{\gamma^{2}}{\eta \rho\left[\frac{G M_{E}(1+e \cos B)^{2}}{a^{2}\left(1-e^{2}\right)^{2}}\right.}\left[\frac{3 G M_{E} R_{E}^{2} J_{2}(1+e \cos B)^{4}\left(\sin ^{2} B \sin ^{2} i-1\right)}{2 a^{4}\left(1-e^{2}\right)^{4}}\right]
\end{aligned}
$$

$$
\left.Q_{2}=-\frac{\left[\begin{array}{l}
-\frac{2 e A \sin B G M_{E}(1+e \cos B)}{a^{2}\left(1-e^{2}\right)^{2}}- \\
+\frac{6 e G M_{E} A J_{2} R_{E}^{2} \sin B\left(\sin ^{2} B \sin ^{2} i-1\right)}{a^{4}\left(1-e^{2}\right)^{4}}
\end{array}\right]}{\left[\frac{G M_{E} J_{2} R_{E}^{2} A \sin 2 B \sin ^{2} i(1+e \cos B)^{4}}{\left.\frac{G M_{E}(1+e \cos B)^{2}}{a^{2}\left(1-e^{2}\right)^{2}}-e^{2}\right)^{4}}\right.}\right]^{2}
$$

Specifically, for circular orbits $e=0$ and $\varphi=0^{\circ}, 45^{\circ}$, $90^{\circ}$ we obtain respectively that Equation 50:

$v=\left[\frac{\gamma^{2}}{36 \rho \eta t^{4}}\left(\frac{6 G M_{E}}{a^{2}}+\frac{3 G M_{E} R_{E}^{2} J_{2}}{2 a^{4}}\right)^{-1}\right]^{1 / 3}$

Next defining Equation 51:

$Q_{3}=\frac{G M_{E}}{a^{2}}-\frac{3 G M_{E} R_{E}^{2} J_{2}}{2 a^{4}}\left(\frac{\sin ^{2}(n t)}{2}-1\right)$

And therefore Equation 52:

$v=\left[\frac{\frac{3 n \gamma^{2} G M_{E} R_{E}^{2} \sin (2 n t) t}{4 a^{4} \rho \eta Q_{3}^{2}}+\frac{\gamma^{2}}{\eta \rho Q_{3}}}{6^{2 / 3}\left(\frac{\gamma^{2} t}{\eta \rho Q_{3}}\right)^{2 / 3}}\right]^{1 / 3}$

And finally Equation 53:

$$
v=\left[\frac{\frac{3 n \gamma^{2} G M_{E} R_{E}^{2} \sin (2 n t) t}{2 a^{4} \rho \eta Q_{3}^{2}}+\frac{\gamma^{2}}{\eta \rho Q_{3}}}{6^{2 / 3}\left(\frac{\gamma^{2} t}{\eta \rho Q_{3}}\right)^{2 / 3}}\right]^{1 / 3}
$$

For elliptical orbits with eccentricity $e \neq 0$ and the same inclinations as above we obtain the following capillary meniscus height velocities when the experiment takes place in orbit and therefore for $i=0^{\circ}$ we obtain Equation 54 and 55: 


$$
\begin{aligned}
& Q_{4}=\frac{G M_{E}(1+e \cos B)^{2}}{a^{2}\left(1-e^{2}\right)^{2}}-\frac{3 G M_{E} R_{E}^{2} J_{2}(1+e \cos B)^{4}}{2 a^{4}\left(1-e^{2}\right)^{4}} \\
& Q_{5}=-\frac{2 e A G M_{E}(1+e \cos B)^{2} \sin B}{a^{2}\left(1-e^{2}\right)^{2}} \\
& -\frac{6 e A G M_{E} R_{E}^{2} J_{2}(1+e \cos B)^{4} \sin B}{2 a^{4}\left(1-e^{2}\right)^{4}}
\end{aligned}
$$

And therefore the meniscus capillary height velocity in an equatorial elliptical orbit becomes Equation 56:

$v_{h(t)}=\frac{\left(\frac{\gamma^{2}}{\eta \rho Q_{4}}\left(1-\frac{Q_{5} t}{Q_{4}}\right)\right)}{6^{2 / 3}\left(\eta \rho Q_{4}\right)^{2 / 3} t^{2 / 3}}$

For the inclination of $45^{\circ}$ we similarly obtain that Equation 57 to 59:

$$
\begin{aligned}
& Q_{6}=\frac{G M_{E}(1+e \cos B)^{2}}{a^{2}\left(1-e^{2}\right)^{2}}- \\
& \frac{3 G M_{E} R_{E}^{2} J_{2}(1+e \cos B)^{4}}{2 a^{4}\left(1-e^{2}\right)^{4}}\left(\frac{\sin ^{2} B}{2}-1\right) \\
& Q_{5}=-\frac{2 e A G M_{E}(1+e \cos B)^{2} \sin B}{a^{2}\left(1-e^{2}\right)^{2}}- \\
& \frac{3 e A G M_{E} R_{E}^{2} J_{2}(1+e \cos B)^{4} \sin 2 B}{4 a^{4}\left(1-e^{2}\right)^{4}} \\
& +\frac{6 A e G M_{E} R_{E}^{2} J_{2} \sin B(1+e \cos B)^{3}}{a^{4}\left(1-e^{2}\right)^{4}}\left(\frac{\sin ^{2} B}{2}-1\right) \\
& v_{h(t)}=\frac{\left(\frac{\gamma^{2}}{\eta \rho Q_{6}}\left(1-\frac{Q_{7} t}{Q_{6}}\right)\right)}{6^{2 / 3}\left(\eta \rho Q_{6}\right)^{2 / 3} t^{2 / 3}}
\end{aligned}
$$

\section{NUMERICAL RESULTS}

Next, for the evaluation of our results we are going to use the following parameters, a cylinder diameter $\mathrm{d}=$ $1030 \mathrm{~mm}$, which implies a capillary radius $r=0.005$ $0.015 \mathrm{~m}$, filled with silicon oil of liquid surface tension $\gamma=20 \mathrm{mNm}^{-1}$ and viscocity $\eta=10-1000 \mathrm{mPas}$ and silicon oil density $\rho=760 \mathrm{~kg} / \mathrm{m}^{3}$ (Ponomarenko et al., 2011). Similarly, for the mass/radius of the Earth we use $M_{E},=5.94 \times 10^{24} \mathrm{~kg}$ and $R_{E}=6378.1363 \mathrm{~km}$, angular velocity of rotation $\omega_{E}=7.292115 \times 10^{-5} \mathrm{rad} / \mathrm{s}$ (Vallado, 2001) and the oblateness coefficient $J_{2}=$ 0.0010827 (Kaula, 2000) and the period of the revolution of the spacecraft at $300 \mathrm{~km}$ is $T_{\text {rev }}=$ $5463.282 \mathrm{~s}$ and its mean motion $n=0.001149492 \mathrm{rad} / \mathrm{s}$. Our results are tabulated in the tables below.

\section{DISCUSSION}

As a first step, Table 1 below presents the numerical results for the meniscus capillary height and for an experiment that takes place on surface of the Earth, using Ponomarenko et al. (2011) result, as well as the corrected acceleration at various geocentric latitudes $\varphi=0^{\circ}, 45^{\circ}$, $90^{\circ}$, in the time interval $t=100 \mathrm{~s}$. Next, in Table 2, we present numerical results Capillary meniscus height for an experiment that takes place on the Earth for $100 \mathrm{~s}$ and at various geocentric latitudes and using our modified gravity result as given by Equation 4 .

Similarly, in Table 3-6 we present capillary height raise for various orbital eccentricities when the particular capillary experiment is taking place aboard an orbiting spacecraft $300 \mathrm{~km}$ above the Earth surface, using Ponomarenko et al. (2011) as well as our corrected gravity results. In Table 7 we demonstrate the change of the meniscus capillary height as a function of spacecraft eccentricity and for the inclination $i=0$. The capillary height has been calculated for a time $t$ equal $100 \mathrm{sec}$ and also $5463.283 \mathrm{~s}$ a time that is equal to the period of the spacecraft revolution around the planetary body at 300 $\mathrm{km}$, Earth in our case. Next, we find that the capillary meniscus height is higher for an experiment that takes place at the equator and lower for experiments at the poles in accordance with Equation 4, since $h(t) \propto g^{-1 / 3}$. Similarly, there is a $9 \%$ difference between the numerical results derived by Ponomarenko's formula and our derived formula with a corrected gravitational acceleration in which the $J_{2}$ harmonic and the angular velocity of the Earth $\omega_{E}$ has been taken into account. On the surface of the Earth, using our corrected gravity we find that $\mathrm{h}_{0}=1.02 \mathrm{~h}_{90}$, where for a 50 times higher viscosity we obtain that $h_{0}\left(\eta_{1}\right)=3.7 h_{0}\left(\eta_{2}\right)$, where $\eta_{1}<$ $\eta_{2}$. Finally, in Table 8. we obtain the capillary meniscus height for an experiment that takes place in a circular orbit of various inclinations at the orbital altitude of 300 $\mathrm{km}$, for the duration of the orbital period of the spacecraft $T=5463.283 \mathrm{~s}$ using our corrected gravity result. We find that circular polar orbits result to a lesser meniscus height raise when compared to the equatorial orbit. As an example in Table 1 we see that for circular equatorial orbits the meniscus height does change in spite the fact that different eccentricities are considered. In other words inclination does not affect the meniscus 
height in circular orbits. This is due to the fact that in circular orbits, orbital acceleration remains the same, as the spacecraft orbits around the Earth.

Table 1. Capillary meniscus height for an experiment that takes place on the Earth for $100 \mathrm{~s}$ and at various geocentric latitudes and the result of Ponomarenko et al. (2011), with $\eta=20$ and $1000 \mathrm{mPs}$ and $\gamma=20 \mathrm{mNm}^{-1}$

\begin{tabular}{lll}
\hline $\begin{array}{l}\text { Geocentric latitude } \\
\varphi^{\circ}\end{array}$ & $\begin{array}{l}\text { Time } \\
t[\mathrm{~s}]\end{array}$ & $\begin{array}{l}\text { Capillary height } \\
h[\mathrm{~mm}]\end{array}$ \\
\hline 0 & 100 & 65.080 \\
45 & & 64.440 \\
90 & & 63.821 \\
0 & 100 & 17.665 \\
45 & & 17.492 \\
90 & & 17.324 \\
\hline
\end{tabular}

Table 2. Capillary meniscus height for an experiment that takes place on the Earth for $100 \mathrm{~s}$ and at various geocentric latitudes and using our corrected gravity result, with $\eta$ $=20$ and $1000 \mathrm{mPs}$ and $\gamma=20 \mathrm{mNm}^{-1}$

\begin{tabular}{llr}
\hline $\begin{array}{l}\text { Geocentric latitude } \\
\varphi^{\circ}\end{array}$ & $\begin{array}{l}\text { Time } \\
t[\mathrm{~s}]\end{array}$ & $\begin{array}{l}\text { Capillary height } \\
h[\mathrm{~mm}]\end{array}$ \\
\hline 0 & 100 & 59.129 \\
45 & & 58.547 \\
90 & & 57.985 \\
0 & 100 & 16.050 \\
45 & & 15.892 \\
90 & & 15.740 \\
\hline
\end{tabular}

Table 3. Capillary meniscus height for an experiment that takes place in an equatorial circular orbit at the orbital altitude of $300 \mathrm{~km}$, in $100 \mathrm{~s}$ and $5463.283 \mathrm{~s}$ using Ponomarenko et al. (2011) result

\begin{tabular}{llr}
\hline & & \multicolumn{2}{c}{ Capillary height } \\
Orbital eccentricity & $e=0$ & $h[\mathrm{~mm}]$ \\
\hline Inclination & $i=0^{\circ}$ & 66.993 \\
& $i=45^{\circ}$ & 66.993 \\
& $i=90^{\circ}$ & 66.993 \\
Inclination & $i=0^{\circ}$ & 254.205 \\
& $i=45^{\circ}$ & 254.205 \\
& $i=90^{\circ}$ & 254.205 \\
\hline
\end{tabular}

Table 4. Capillary meniscus height for an experiment that takes place in an equatorial circular orbit at the orbital altitude of $300 \mathrm{~km}$, in $100 \mathrm{~s}$ and $5463.283 \mathrm{~s}$ using our corrected gravity result

\begin{tabular}{lr}
\hline Orbital eccentricity & \multicolumn{2}{c}{ Capillary height } \\
$e=0$ & $h[\mathrm{~mm}]$ \\
\hline Inclination $i=0^{\circ}$ & 60.863 \\
$i=45^{\circ}$ & 60.863 \\
$i=90^{\circ}$ & 60.863 \\
Inclination $i=0^{\circ}$ & 230.945 \\
$i=45^{\circ}$ & 230.945 \\
$i=90^{\circ}$ & 230.945 \\
\hline
\end{tabular}

Table 5. Capillary meniscus height for an experiment that takes place in an elliptical orbit at the orbital altitude of $300 \mathrm{~km}$, in $100 \mathrm{~s}$ and $5463.283 \mathrm{~s}$ using Ponomarenko et al. (2011) result

\begin{tabular}{ll}
\hline Orbital eccentricity & Capillary height \\
$e=0.1$ & $h[\mathrm{~mm}]$ \\
\hline Inclination $i=0^{\circ}$ & 66.552 \\
$i=45^{\circ}$ & 66.050 \\
$i=90^{\circ}$ & 65.562 \\
Inclination $i=0^{\circ}$ & 252.533 \\
$i=45^{\circ}$ & 250.624 \\
$i=90^{\circ}$ & 248.772 \\
\hline
\end{tabular}

Table 6. Capillary meniscus height for an experiment that takes place in an equatorial circular orbit at the orbital altitude of $300 \mathrm{~km}$, in $100 \mathrm{~s}$ and $5463.283 \mathrm{~s}$ using our corrected gravity result

\begin{tabular}{lr}
\hline Orbital eccentricity & \multicolumn{2}{c}{ Capillary height } \\
$e=0.1$ & $h[\mathrm{~mm}]$ \\
\hline Inclination $i=0^{\circ}$ & 60.470 \\
$i=45^{\circ}$ & 60.010 \\
$i=90^{\circ}$ & 59.567 \\
Inclination $i=0^{\circ}$ & 229.441 \\
$i=45^{\circ}$ & 227.707 \\
$i=90^{\circ}$ & 226.024 \\
\hline
\end{tabular}

Table 7. Capillary meniscus height for an experiment that takes place in an equatorial orbits of various eccentricities at the orbital altitude of $300 \mathrm{~km}$, for the duration of the orbital period of the spacecraft $T=5463.283 \mathrm{~s}$ using our corrected gravity result

\begin{tabular}{lr}
\hline $\begin{array}{l}\text { Orbital eccentricity } \\
\text { Inclination } i=0\end{array}$ & $\begin{array}{c}\text { Capillary height } \\
h[\mathrm{~mm}]\end{array}$ \\
\hline Eccentricity $e=0.0$ & 230.960 \\
$e=0.1$ & 229.441 \\
$e=0.2$ & 224.855 \\
$e=0.3$ & 217.112 \\
$e=0.4$ & 206.048 \\
$e=0.5$ & 191.403 \\
$e=0.6$ & 172.800 \\
$e=0.7$ & 149.600 \\
$e=0.8$ & 121.102 \\
$e=0.9$ & 90.577 \\
\hline
\end{tabular}

Table 8. Capillary meniscus height for an experiment that takes place in a circular orbit of various inclinations at the orbital altitude of $300 \mathrm{~km}$, for the duration of the orbital period of the spacecraft $\mathrm{T}=5463.283 \mathrm{~s}$ using our corrected gravity result

\begin{tabular}{lr}
\hline $\begin{array}{l}\text { Orbital eccentricity } \\
e=0\end{array}$ & $\begin{array}{c}\text { Capillary height } \\
\text { velocity } v[\mathrm{~mm} / \mathrm{s}]\end{array}$ \\
\hline Inclination $i=0^{\circ}$ & 0.014092 \\
$i=45^{\circ}$ & 0.014016 \\
$i=90^{\circ}$ & 0.013942 \\
\hline
\end{tabular}


Since we are interested in experiments that take place in spacecrafts in orbit, we have calculated all the corresponding meniscus heights for one orbital revolution period at the orbital altitude of the spacecraft, i.e., $t=T=5463.282 \mathrm{~s}$.

Fig. 1 gives demonstrates the wetting effect between two solid rods of the same or different sizes. For the same elapsed time, high viscosity fluids result to significantly smaller meniscus heights. Similarly, as the eccentricity of an orbit of certain inclination increases the corresponding meniscus height decreases drastically, for any given time of observation, with equatorial circular orbits resulting to the smaller decrease. In Fig. 2, we plot the meniscus height as a function of geocentric latitude, which demonstrates a maximum height at the equatorial latitude of zero degrees. In Fig. 3, we give a 3-D plot of the capillary meniscus height as a function of time $t$ and the acceleration of gravity $g$ for an experiment that takes place on the surface Earth using the result obtained by Ponomarenko et al. (2011), where in Fig. 4 represents a similar plot using the result derived by the authors of this study. A high meniscus height requires a small gravitational acceleration and a higher interaction time and we find that $h_{\text {cor }}=0.422 h_{\text {Pon }}\left(\frac{t}{g}\right)^{-2 / 3}$, where $\mathrm{h}_{\text {cor }}$ is the meniscus height derived by our corrected gravity and $h_{P o n}$ is the meniscus height calculated by Ponomarenko's result. Next, in Fig. 5 we give a 3-D plots of the capillary meniscus height as a function of time $t$ and the mass of the spherical body $M$ on the surface of which the experiment that takes place using the result derived by the authors of this study, where Fig. 6 plots the meniscus height as a function of viscosity $\eta$ and fluid surface tension $\gamma$. Furthermore, for the time of a full orbital period we find the following ratio between the height of capillary for an experiment taking place in a circular equatorial orbit, to that taking place in a highly elliptical orbit of eccentricity $e=0.9$, i.e., $h_{\text {cir }}=2.55 h_{\text {ell }}$.
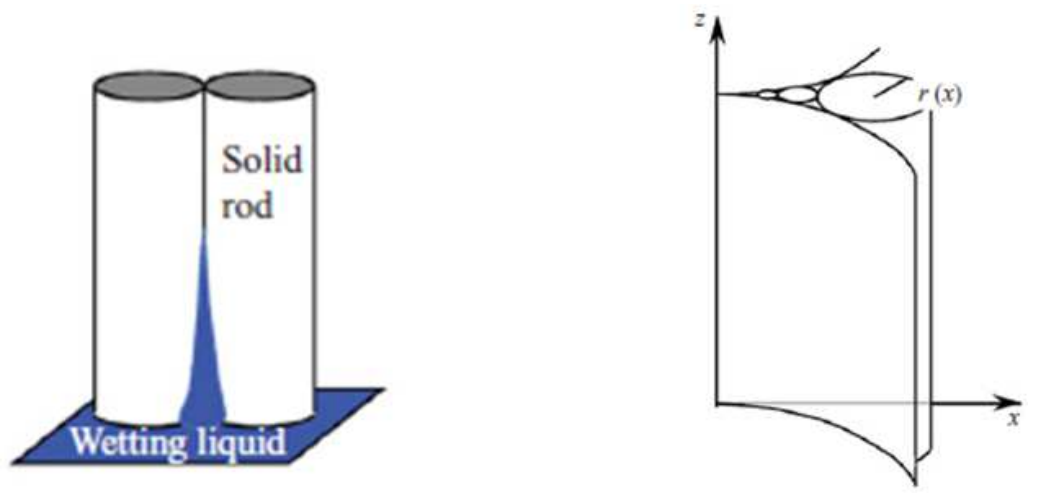

Fig. 1. Wetting effect: (Ponomarenko et al., 2011)

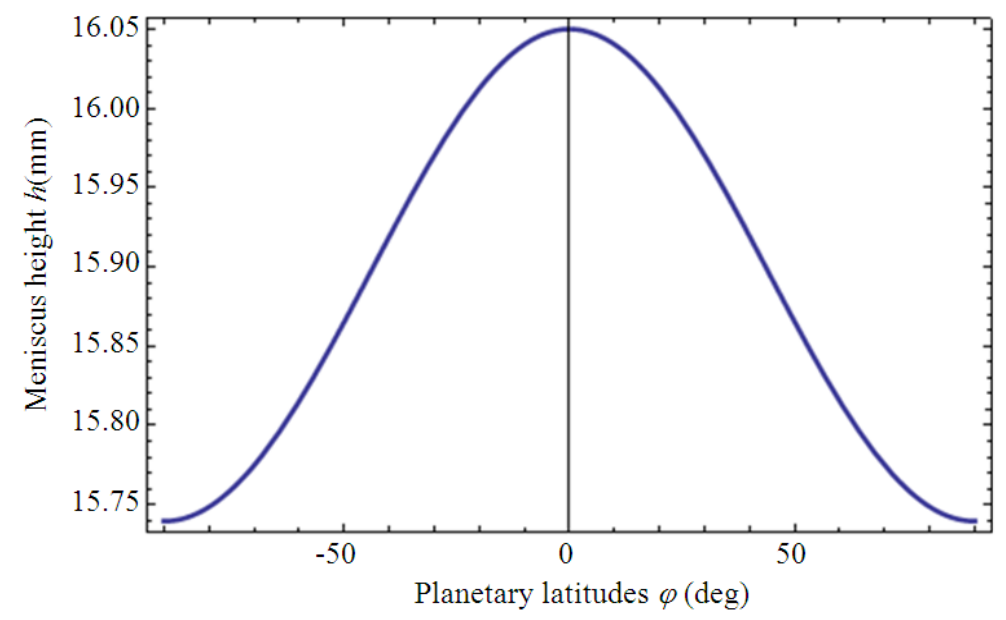

Fig. 2. Plot of meniscus height as a function of geocentric latitude 


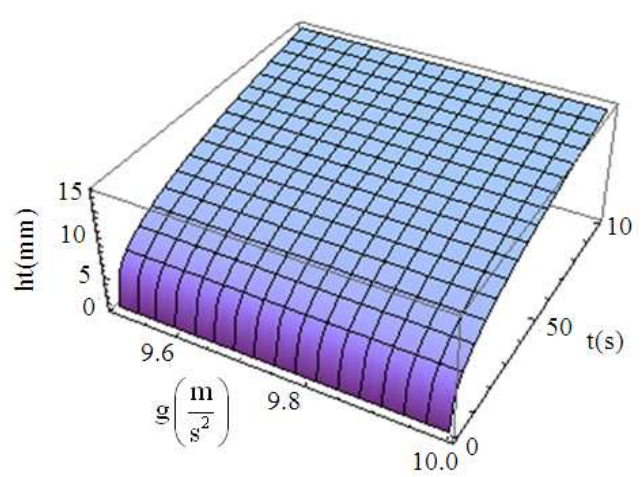

Fig 3. 3-D plot of the capillary meniscus height as a function of time $t$ and acceleration of gravity $g$ for an experiment that takes place on the Earth using the result by Ponomareko et al. (2011)

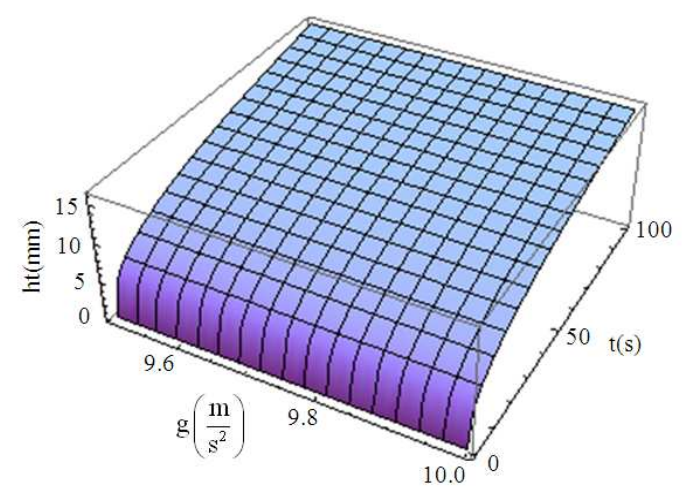

Fig 4. 3-D plot of the capillary meniscus height as a function of time $t$ and acceleration of gravity $g$ for an experiment that takes place on the Earth by the authors of this study

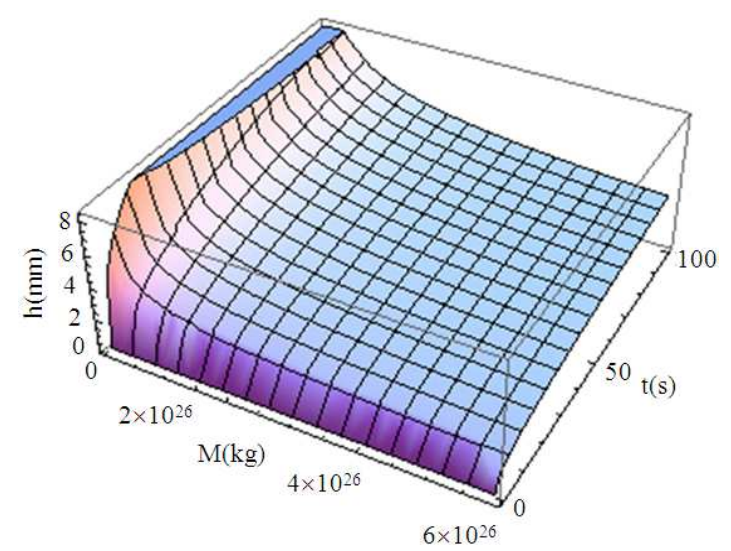

Fig. 5. 3-D plot of the capillary meniscus height as a function of time $t$ and the mass of the spherical body $\mathrm{M}$ on the surface of which the experiment that takes place using the result derived by the authors of this study

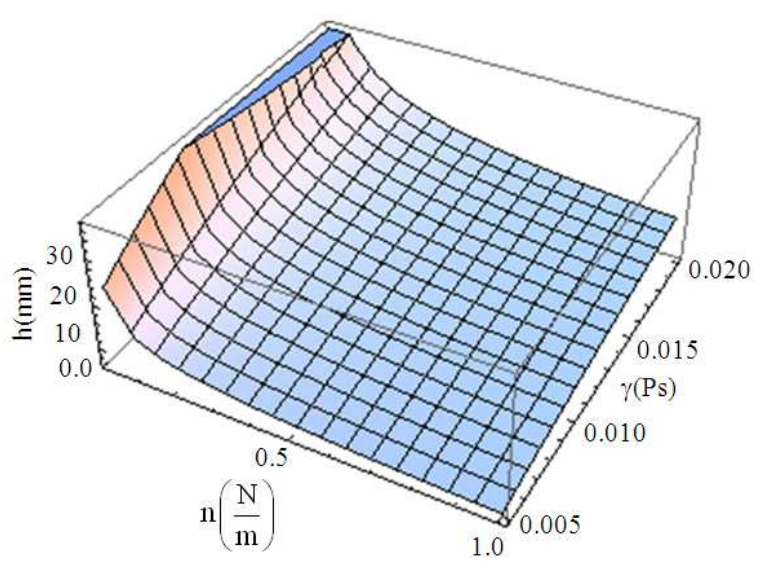

Fig. 6. 3-D plot of the capillary meniscus height as a function of time $t$ and the mass of the spherical body $\mathrm{M}$ on the surface of which the experiment that takes place using the result derived in this study

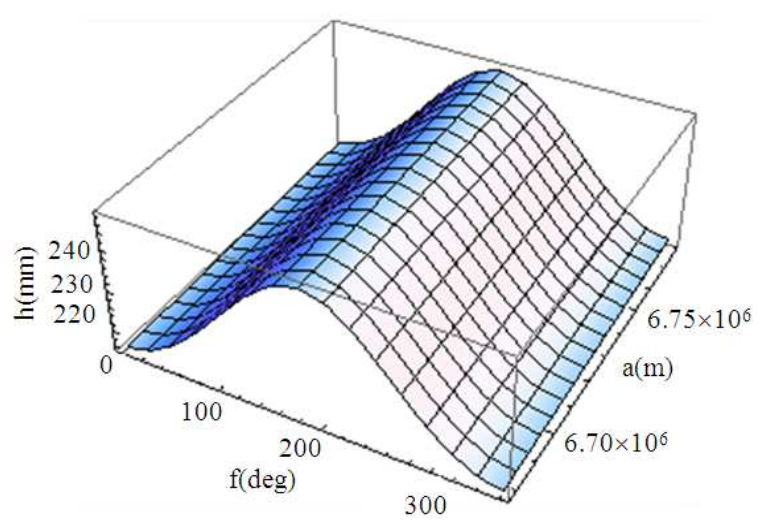

Fig. 7. 3-D plot of the capillary meniscus height as a function of semi major axis $a$ and true anomaly $f$, of the orbit for an experiment in an elliptical orbit with eccentricity $e=0.1$, taking place aboard an orbiting spacecraft using the result derived in this study for a time $t$ equals to the spacecraft's orbital period

In relation to the capillary height time rate of change, we find that laboratory sites at the equator will exhibit larges time rates of change for the meniscus height. Similarly, for experiments above a spacecraft we find that circular equatorial orbits exhibit the highest time rates of change, where elliptical orbits exhibit smaller time that reduce as the eccentricity increases. Since capillary experiments are major part Earth based experiments as well as of the research taking place above the International Space Station (ISS) (Weislogel et al., 2008), our work can prove to be essential in high accuracy experiments, in which the inclusion of the Earth's rotation as well as the $J_{2}$ will be required (Fig. 7-9). 


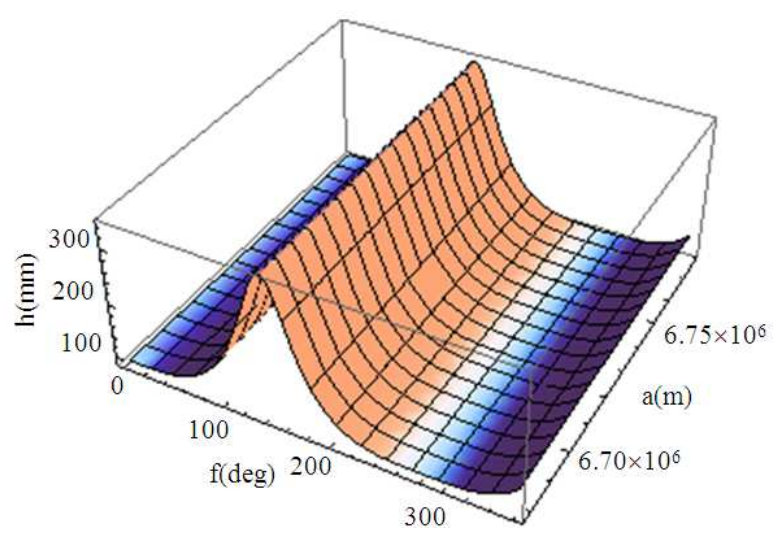

Fig. 8. 3-D plot of the capillary meniscus height as a function of semi major axis $a$ and true anomaly $f$, of the orbit for an experiment in an highly elliptical orbit with eccentricity $e=0.83$, taking place aboard an orbiting spacecraft using the result derived in this study, for a time $t$ equals to the spacecraft's orbital period

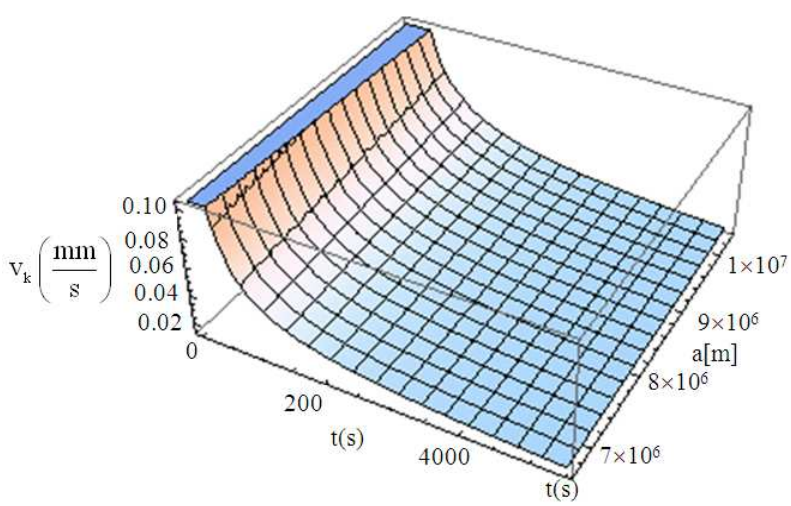

Fig. 9. 3-D plot of the capillary meniscus velocity as a function of semi major axis $a$ and time $t$, of the orbit for an experiment in an circular orbit with eccentricity $e=0$, taking place aboard an orbiting spacecraft using the result derived in this study, for a time $t$ equals to the spacecraft's orbital period

In Fig. 7 we plot the meniscus height as a function of the spacecraft's semimajor axis and true anomaly, orbiting in an elliptical orbit an eccentricity $e=0.1$. We find that the height $h$ exhibits an increasing behaviour that reaches a maximum at the value of the true anomaly $f=180^{\circ}$ and then progressively declines. In Fig. 8, a highly eccentric orbit i.e., $e=0.85$ results in a sharper but compressed peak for the meniscus height exhibits a maximum around $f=140^{\circ}$, that decreases asymmetrically with respect to the central peak.

\section{CONCLUSION}

In this study we have considered the capillary rise of wetting in liquids in the corners. For, that we have examine and extended Ponomarenko et al. (2011) result by taking into account a term that Ponomareko omits in the derivation of his result. We find that the inclusion of this term results in the following relation i.e., $h_{\text {new }}(t) \cong 0.9085 h_{\text {Pon }}(t)$. In particular and on the surface of the Earth we have corrected the gravitational acceleration for the oblateness coefficient $J_{2}$ and also for the Earth's rotation via its angular velocity $\omega_{E}$. We father extent the meniscus height in the case a wetting experiment takes place above and orbiting spacecraft by writing the gravitational acceleration $g$ as a function of the satellites orbital elements. We find that on the surface of the Earth experiments at the equator result to higher capillary heights, where experiments on the poles result in lower capillary heights. Similarly, equatorial circular orbits result to higher capillary heights when compared to elliptical orbits of various eccentricities. Highly elliptical equatorial orbits considerably reduce the capillary height, when compared with the capillary height resulting from equatorial circular orbits. These observations are suggestive of implications in biological systems where capillary formations are integral for their growth and function. Water transport and storage in plants depends strongly in the capillary action of xylem formations, the finding that capillary height varies significantly from the pole to the equator may have implications on the flora distributed in a range of geographic latitudes. For example, it may be a favorable factor for the growth, development and sustainability of gigantic plants such as the trees found in the tropics. More over, capillary-driven root module designs for growing plants in microgravity which require minimal external control have been proposed (Jones, 1999). Matching growth media and water retention characteristics to the porous membrane characteristics is essential for supplying adequate liquid flux and gas exchange (Jones, 1998). The dependence of capillary height in the flight path suggests testing of these materials in different orbital conditions in order to achieve functional adjustments.

\section{REFERENCES}

Ferrero, F., 2003. Wettability measurements on plasma treated synthetic fabrics by capillary rise method. Polymer Test., 22: 571-578. DOI: 10.1016/S01429418(02)00153-8 
Galet, L., S. Patry and J. Dodds, 2010. Determination of the wettability of powders by the washburn capillary rise method with bed preparation by a centrifugal packing technique. J. Colloid Interface Sci., 346: 470-475. DOI: 10.1016/j.jcis.2010.02.051

Jones, S.B., 1998. A capillary-driven root module for plant growth in microgravity. Adv. Space Res., 22: 1407-12. DOI: 10.1016/S0273-1177(98)00215-4

Jones, B.S., 1999. Microgravity effects on water flow and distribution in unsaturated porous media: Analyses of flight experiments. Water Resources Res., 35: 929-929. DOI: 10.1029/1998WR900091

Hoffman, R.L., 1983. A study of the advancing interface.2. Theoretical prediction of the dynamic contact angle in liquid-gas systems. J. Rheol., 94: 470-486. DOI: 10.1016/0021-9797(83)90287-4

Haranas, I. and M. Harney, 2008. Detection of the relativistic corrections to the gravitational potential using a sagnac interferometer. Progress Phys., 3: 38.

Haranas, I., I., Gkigkitzis and G.D. Zouganelis, 2012. g Dependent particle concentration due to sedimentation. Astrophys. Space Sci., 342: 31-43. DOI: 10.1007/s10509-012-1151-1

Karoglou, M., A. Moropoulou, A. Giakoumaki and M.K. Krokida, 2005. Capillary rise kinetics of some building materials. J. Colloid Interface Sci., 284: 260-264. DOI: 10.1016/j.jcis.2004.09.065

Kaneki, Y., Y. Sasaki, K. Ishii and M. Iguchi, 2000. Rate of capillary rise in the porous media under microgravity. ISIJ Int.

Kaula, W., 2000. Satellite Geodesy. 1st Edn., Dover Publication.

Marmur, A., 2003. Kinetics of penetration into uniform porous media: Testing the equivalent-capillary concept. Langmuir, 19: 5956-5959. DOI: 10.1021/la034490v
Murray, C.D. and S.F. Dermott, 1999. Solar System Dynamics. 1st Edn., Cambridge University Press, Cambridge, ISBN-10: 0521575974. pp: 41.

Nobel, P.S., 1999. Physicochemical and Environmental Plant Physiology. 4th Edn., Academic Press, Burlington, ISBN-10: 0080920896 pp: 600.

Ostrach, S., 1982. Low-gravity fluid-flows. Annual Rev. Fluid Mechan., 14: 313-345. DOI: 10.1146/annurev.fl.14.010182.001525

Ponomarenko, A., D. Quere and C. Clanet, 2011. A universal law for capillary rise in corners. J. Fluid Mechan., 666: 146-154. DOI: 10.1017/S0022112010005276

Schramm, L.L., D. Hart, F. Wassmuth, J.C. Legros and E.N. Smirnov et al., 2003. Capillary flow in porous media under highly reduced gravity investigated through high altitude parabolic aircraft flightsand NASA space shuttle flight. Petroleum Society.

Smirnov, N.N., V.F. Nikitin, J.C. Legros, E. Istasse and L. Schramm et al., 2004. Microgravity investigations of capillary-driven imbibition and drainage in inhomogeneous porous media. Acta Astronaut., 54: 39-52. DOI: 10.1016/S00945765(02)00278-3

Tanner, L.H., 1979. Spreading of silicone oil drops on horizontal surfaces. J. Phys. D-Applied Phys., 12: 1473-1473. DOI: 10.1088/0022-3727/12/9/009

Vallado, D.A., 2001, Fundamentals of Astrodynamics and Applications. 1st Edn., Springer, Dordrecht, ISBN-10: 0792369033, pp: 958.

Weislogel, M., R. Jenson, Y. Chen, S. Collicott and M. Dreyer et al., 2008. Postflight summary of the capillary flow experiments aboard the international spacestation. Proceedings of the 59th International Astronautical Congress, Sept. 29Oct. 3, Glasgow, United Kingdom. 